\title{
Infecção odontogênica grave após exodontia de terceiros molares - relato de caso
}

\author{
Severe odontogenic infection after third molar extraction - case report \\ Infección odontogénica grave después de la extracción del tercer molar - reporte de caso
}

\section{Resumo}

As infecções odontogênicas são complicações comumente enfrentadas pelos dentistas pós-exodontia de terceiros molares, dessarte, é de suma importância que os cirurgiões maxilofaciais saibam diagnosticar e tratar corretamente esses casos. Assim, o objetivo é relatar um caso clínico de paciente mulher de 20 anos de idade com infecção odontogênica grave. Paciente leucoderma, com aumento volumétrico extenso em face a direita, histórico de exodontia prévia em elemento dental com quadro de pericoronarite prévia a extração, admitida pelo Serviço de Cirurgia e Traumatologia Bucomaxilofacial do Hospital Universitário Maria Aparecida Pedrossian. Edema extenso em região submandibular a direita, dispneia, disfagia e trismo acentuado. Aos exames de imagem, ausência dos dentes 18 e 48 e lojas de coleção purulenta. O tratamento foi cirúrgico com drenagem do exsudato, curetagem das regiões de exodontia e antibioticoterapia. Associado ao procedimento cirúrgico foram solicitadas avaliação de especialidades médicas para controle sistêmico da paciente. Após 7 dias de internação a paciente recebeu alta, mas manteve retornos pósoperatórios. O quadro severo apresentado pela paciente mostra a magnitude que pode atingir a infecção odontogênica. O tratamento cirúrgico pós-operatório corroborado com antibioticoterapia e controle sistêmico da paciente foram decisivos. O tratamento da pericoronarite antes da exodontia é o fator decisivo para se evitar processos infecciosos da magnitude apresentada pela paciente.

Palavras-chave: Dente serotino; Complicações pós-operatórias; Infecção focal dentária; Cirurgia bucal.

\begin{abstract}
Odontogenic infections are complications commonly faced by dentists after third molar extraction, thus, it is extremely important that maxillofacial surgeons know how to correctly diagnose and treat these cases. Thus, the objective is to report a clinical case of a 20 -year-old female patient with severe odontogenic infection. Leukoderma patient, with extensive volumetric increase on the right side, history of previous extraction of a dental element with a picture of pericoronitis prior to extraction, admitted by the Maxillofacial Surgery and Traumatology Service of Hospital Universitário Maria Aparecida Pedrossian. Extensive edema in the right submandibular region, dyspnea, dysphagia, and severe trismus. On imaging exams, absence of teeth 18 and 48 and purulent collection stores. Treatment was surgical with drainage of the exudate, curettage of the areas of extraction and antibiotic therapy. Associated with the surgical procedure, an evaluation of medical specialties for systemic control of the patient was requested. After 7 days of hospitalization, the patient was discharged, but she continued to return postoperatively. The severe picture presented by the patient shows the magnitude that the odontogenic infection can reach. Postoperative surgical treatment corroborated with antibiotic therapy and systemic control of the patient were decisive. The treatment of pericoronitis before extraction is the decisive factor to avoid infectious processes of the magnitude presented by the patient.
\end{abstract}

Keywords: Molar, third; Postoperative complications; Focal infection, dental; Surgery, oral. 


\begin{abstract}
Resumen
Las infecciones odontogénicas son complicaciones que comúnmente enfrentan los dentistas después de la extracción del tercer molar, por lo que es extremadamente importante que los cirujanos maxilofaciales sepan cómo diagnosticar y tratar correctamente estos casos. Así, el objetivo es reportar un caso clínico de una paciente de 20 años con infección odontogénica severa. Paciente con leucodermia, con aumento volumétrico extenso en el lado derecho, antecedente de extracción previa de un elemento dentario con cuadro de pericoronitis previa a la extracción, ingresado por el Servicio de Cirugía y Traumatología Maxilofacial del Hospital Universitário Maria Aparecida Pedrossian. Edema extenso en región submandibular derecha, disnea, disfagia y trismo severo. En los exámenes de imagen, ausencia de los dientes 18 y 48 y depósitos de colección purulentos. El tratamiento fue quirúrgico con drenaje del exudado, legrado de las zonas de extracción y antibioterapia. Asociado al procedimiento quirúrgico, se solicitó una evaluación de especialidades médicas para el control sistémico del paciente. Luego de 7 días de internación, la paciente fue dada de alta, pero continuó regresando postoperatoriamente. El cuadro severo que presenta el paciente muestra la magnitud que puede alcanzar la infección odontogénica. El tratamiento quirúrgico postoperatorio corroborado con antibioterapia y el control sistémico del paciente fueron determinantes. El tratamiento de la pericoronitis previo a la extracción dentaria es el factor decisivo para evitar procesos infecciosos de la magnitud que presenta el paciente.
\end{abstract}

Palabras clave: Tercer molar; Complicaciones postoperatorias; Infección focal dental; Cirugía oral.

\title{
1. Introdução
}

As extrações dentárias são procedimentos frequentes nos consultórios odontológicos, rotineiramente geram apenas um desconforto passageiro de rápida cicatrização, entretanto, não é rara a ocorrência de complicações infecciosas pós-exodontias. Essas condições são vistas principalmente após a extração dos terceiros molares, tanto pela complexidade dos procedimentos operatórios, devido à localização e posição desses dentes, quanto pela chance de apresentarem condições pré-operatórias desfavoráveis, predisponentes às infecções, se não tratadas previamente (Akinbami \& Godspower, 2014).

As infecções odontogênicas podem ser causadas por cáries extensas que atingem a polpa dental e o periápice do mesmo e assim geram complicações endodônticas, periodontites agressivas e pericoronarites não tratadas (Ogle, 2017). No tocante a pericoronarite especificamente, é uma infecção do tecido gengival que recobre parcialmente os dentes em erupção sendo os terceiros molares os mais acometidos. Esse estado cria um mucoperiósteo suprajacente que é um arcabouço para alimentos e detritos, nos quais bactérias se multiplicam rapidamente e podem liberar toxinas. Essa condição impede que os dentes sejam extraídos imediatamente, necessitando ser tratada antes da intervenção cirúrgica, uma vez que, é um forte predisponente a infeção odontogênica pós-exodontia. O tratamento varia desde bochechos e irrigações com soro fisiológico, agua oxigenada, solução de iodóforos até à antibioticoterapia (Galvão et al., 2019, Wehr et al., 2019).

As infecções odontogênicas representam, em inúmeros casos, difícil resolução pelo cirurgião bucomaxilofacial, devido a imprevisibilidade da evolução em cada paciente, resultado das condições prévias e sistêmicas de cada um. São divididas em locais ou extensas, a depender do alastramento da infecção aos tecidos adjacentes e espaços fasciais da região da cabeça e pescoço (Brito et al., 2017). Comumente, manifestam-se através de edema, dor localizada acompanhada de inchaço, vermelhidão, presença de trismo e alterações funcionais, como: disfagia, dislalia e dispneia, além de febre e prostração. Podendo evoluir com abscessos localizados, celulites faciais e cervicais, abscessos mediastinais, cerebrais, trombose de seios cavernosos e até sepse, com potencial para levar o paciente a óbito (Pitekova et al., 2010; Ogle, 2017)

A gravidade dessas infecções está relacionada a diversos fatores, como a demora na busca de tratamento pelo paciente, equívocos de diagnóstico ou conduta inicial inadequada, antibioticoterapia empírica, localização topográfica da infecção, estado nutricional do paciente, condições sistêmicas imunossupressoras ou comorbidades, hábitos nocivos, seleção e malignidade dos microrganismos (Velasco \& Soto, 2012). Posto isso, o diagnóstico preciso da infecção odontogênica necessita de uma análise detalhada do caso clínico incluindo exames laboratoriais, histórico médico e odontológico do paciente, métodos de imagem como ultrassonografias, radiografias planas convencionais e tomografias computadorizadas. Ademais, descobrir a causa pode determinar a severidade da infecção (Friedman, 2007; Lima et al., 2018). 
$\mathrm{O}$ tratamento das infecções requer um tratamento urgente que envolva um diagnóstico correto, remoção imediata ou secundária da causa, drenagem cirúrgica e antibioticoterapia, com o objetivo de prevenir uma maior disseminação, além de propiciar ao organismo melhores condições de reparo tecidual (Faverani et al., 2020). Dessa forma, o objetivo desse estudo é relatar um caso de infecção odontogênica grave de origem pericoronária, discutindo seu diagnóstico e tratamento.

\section{Metodologia e Relato de Caso}

O presente caso trata-se de um relato com fins descritivos, exploratórios e com abordagem qualitativa, o qual possui a autorização do paciente por meio do Termo de Consentimento Livre e Esclarecido (TCLE), a fim de mostrar sua relevância clínica e facilitar pesquisas e novos relatos com a mesma temática, sempre baseada em evidência, usando como base referencial Pereira (2018) e Souza et al., (2021).

Paciente de 20 anos de idade, sexo feminino, graduanda em Odontologia, com histórico de anemia ferropriva, imunossuprimida, procurou o Hospital Universitário Maria Aparecida Pedrossian da Universidade Federal de Mato Grosso de Sul queixando-se de dor local, limitação severa de abertura bucal e aumento de volume de grande proporção em região submandibular direita. A paciente apresentava-se sem sinais de febre, com quadro de diarreia e episódios de êmese. Além disso, a mesma relatou ter sido submetida a procedimento de exodontia, dos elementos 18 e 48, no dia anterior e segundo a mesma, acadêmica de odontologia, não teve intercorrências.

Em pós-operatório imediato recebeu prescrição de analgésico, dipirona 500mg de 6 em 6 horas por 2 dias, antisséptico, digluconato de clorexidina $0,12 \%$ para bochecho por 7 dias e sugestões para não realizar esforços físicos durante 5 dias e fazer uso de compressas frias na região das extrações.

Após 24 horas das exodontias a paciente evoluiu com um edema importante em região submandibular e bucal direita, limitação de abertura bucal e episódios de êmese. Depois de ser submetida ao exame clínico hospitalar, constatou-se que a paciente se encontrava em quadro inicial de celulite facial, apresentando sinais e sintomas de sepse.

Imediatamente a paciente foi admitida pelo serviço de Cirurgia e Traumatologia Bucomaxilofacial do HUMAP, a primeira conduta foi iniciar o tratamento com antibioticoterapia empírica, analgesia e hidratação endovenosa para tentar impedir a progressão e alastramento da infecção. Iniciou-se então a administração endovenosa de clindamicina $600 \mathrm{mg}$ de $8 \mathrm{em}$ 8 horas, ceftriaxona $1 \mathrm{~g}$ de $12 \mathrm{em} 12$ horas, tramadol 50mg de 6 em 6 horas e dipirona $1 \mathrm{~g} \mathrm{em}$ episódios de febre. Além das medicações foi iniciada a terapia de apoio com soro glicosado 15 gotas por minuto.

Foram feitos exames laboratoriais e de imagem para confirmar o diagnóstico. O hemograma mostrou alta taxa de segmentados 15.226,90 mm³ e proteína C-reativa (PCR) elevada 326,36mg/L confirmando o quadro de infecção. A tomografia computadorizada dos seios da face mostrou espessamento e densificação de partes moles na região maxilar e mandibular à direita, associado a formação hipoatenuante e mal definida de permeio ao subcutâneo (sugestivo de coleção purulenta), além desta formação, no recesso alveolar do seio maxilar esquerdo, medindo cerca de 2,0 $\mathrm{cm}$, podendo corresponder à cisto de retenção. Além disso, fino espessamento mucoso/cisto de retenção no seio maxilar direito e linfonodos cervicais na região súpero-lateral direita proeminentes. Os demais seios paranasais com transparência preservada, infundíbulos, unidades ostiomeatais e recesso esfeno-etmoidais livres. Cornetos de contornos e dimensões normais.

Vinte e quatro horas após, a paciente ainda não apresentava melhoras no quadro infeccioso e foi realizada a drenagem cirúrgica da coleção purulenta. A cirurgia de drenagem foi realizada com a paciente sob anestesia geral. Após a indução anestésica e intubação orotraqueal, realizou-se a antissepsia extra e intraoral com clorexidina $2 \%$. Infiltração anestésica com lidocaína 2\% associada a epinefrina 1:100.000 em região submandibular direita. Incisão em pele, de aproximadamente 30mm, em região submandibular direita e divulsão por planos até atingir osso mandibular. Colocação de tampão orofaríngeo. Incisão 
intraoral, em mucosa de região de fundo de vestíbulo maxilar posterior direito, drenagem com instrumento rombo em fundo de vestíbulo maxilar posterior direito. Desbridamento e remoção de coágulo e tecido necrótico em região retro molar direita. Irrigação com soro fisiológico. Instalação de dreno de Pen Rose em região de fundo de vestíbulo maxilar direito + sutura com fio de nylon 5.0. Instalação de dreno de Pen Rose em região submandibular direita. Após o procedimento cirúrgico foi realizado acesso venoso central em veia subclávia direita (Figura 1).

Figura 1: Paciente sob anestesia geral e intubação orotraqueal. Aspectos intra e extrabucal da paciente. Edema extenso em hemiface direita. Desbridamento e instalação de dreno de Pen Rose. Confecção de acesso venoso em subclávia direita.
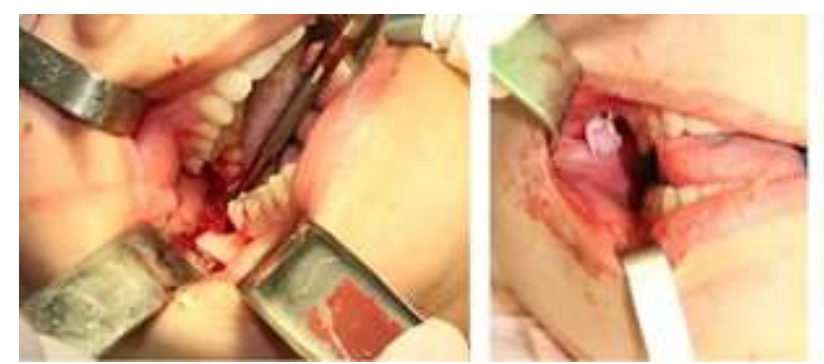
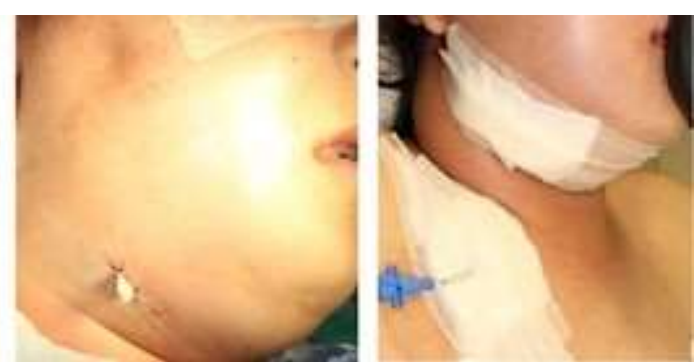

Fonte: Autores.

O conteúdo cirúrgico foi levado para realização de cultura. Com o resultado da cultura, o diagnóstico estabelecido foi de infecção odontogênica grave causada pelo Streptococcus pyogenes. Os antibióticos, analgésicos e a hidratação intravenosa foram mantidas por todo o período de internação, que durou 6 dias. A paciente evoluiu com melhora do quadro infeccioso, melhora gradativa dos exames bioquímicos e da hipotensão.

Após 6 dias de internação a paciente apresentou-se lúcida, orientada em tempo e espaço (LOTE), bem-estar geral (BEG), bom estado nutricional (BEN) e acianótica, afebril e anictérica (AAA), deambulando e verbalizando normalmente, sem queixas álgicas e com ausência de drenagem de exsudato purulento. Diante da evolução, a paciente recebeu alta no mesmo dia com remoção dos drenos de Pen Rose, orientações pós-operatórias, medicações e retorno ambulatorial.

Em retorno pós-operatório de nove dias a paciente apresentava quadro de drenagem de exsudato purulento intraoral e pela incisão cirúrgica associado a disfagia e queixas álgicas. A mesma foi novamente admitida no hospital para a realização de exames. Foram realizados ultrassonografia, bioquímicos e tomografia para investigação do quadro de infecção e administração endovenosa de dipirona 500mg de $6 \mathrm{em} 6$ horas e tramadol 50mg de $8 \mathrm{em} 8$ horas.

O ultrassom exibiu área de aumento da espessura e diminuição da ecogenicidade do tecido subcutâneo à direita, sem a delimitação de coleções líquidas, não apresentando nenhuma loja de coleção purulenta, compatível com edema subcutâneo. Ao exame tomográfico embora com estruturas dentro da normalidade, é evidente a assimetria às custas de grande edema (Figura 2). Aos exames bioquímicos ocorreram alterações de leucopenia e anemia e a paciente foi instruída a procurar atendimento especialista de Hematologia para avaliação do quadro. 
Figura 2: Tomografia computadorizada em reconstruções axial e coronal com janelas para tecido ósseo e tecido mole, respectivamente. Evidencia-se a assimetria bastante evidente de face e a presença de alvéolo do dente 48.
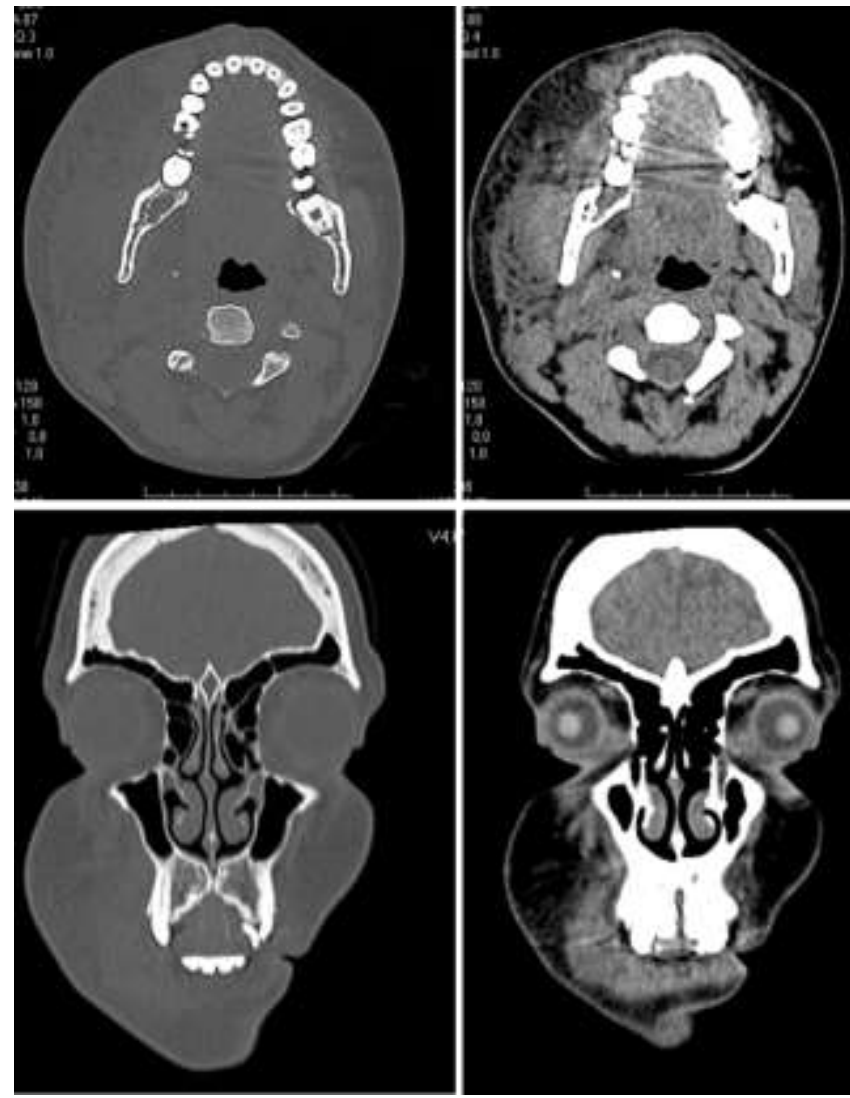

Fonte: Autores.

Em 48 horas de internação a paciente evoluiu novamente para LOTE, BEM, BEG, AAA, deambulando e verbalizando normalmente, diurese presente, sem queixas álgicas e recebeu alta hospitalar com retorno agendado. No dia seguinte a alta do HUMAP, a paciente foi avaliada pela hematologia do Hospital Regional Rosa Pedrossian quanto a leucopenia, e logo apresentou melhora do quadro clínico, sem queixas álgicas, sem sinais flogísticos, evoluindo favoravelmente.

A paciente evoluiu com parestesia no trajeto do nervo lingual a direita até os seis meses de pós-operatório. Hoje evolui para a cura completa da infecção com remissão total do quadro e sem recorrência e/ou demais complicações (Figura 3).

Figura 3: Aspecto clínico após completa remissão do quadro infeccioso. Simetria facial, regressão completa do edema e discreta cicatriz em região do local de drenagem do exsudato purulento.
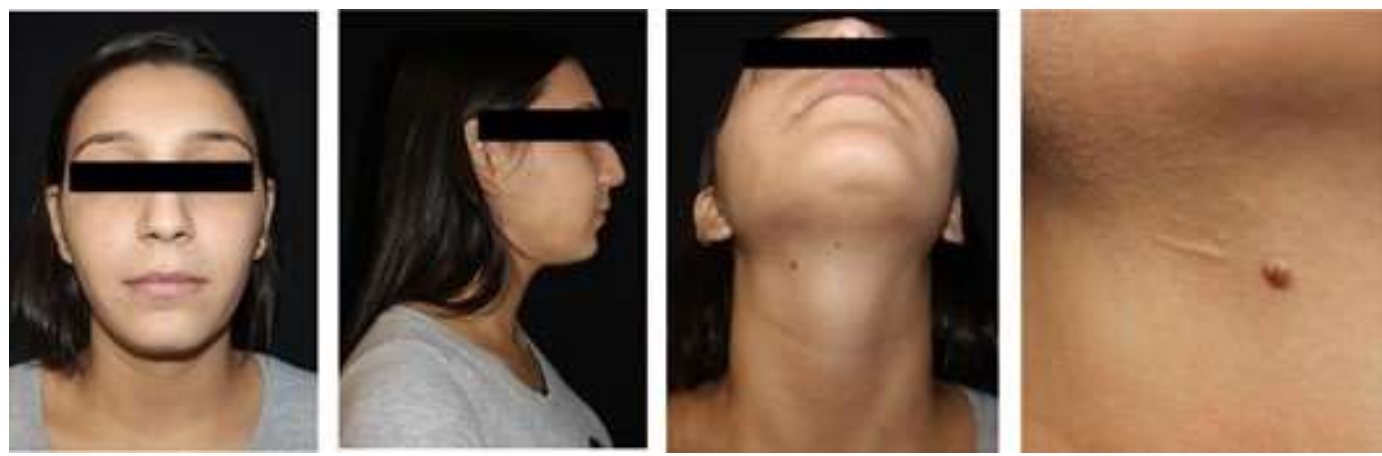

Fonte: Autores. 


\section{Discussão}

As infecções odontogênicas não acometem apenas um sexo ou classe social ou mesmo uma idade pré-estabelecida. Elas são verdadeiros problemas de saúde pública; envolvem todas as categorias, necessitam de um atendimento especializado e por vezes hospitalar, equipamentos e equipe de apoio para a resolução dos casos. Por outro lado, mesmo não fazendo distinção de grupos, indivíduos jovens e do sexo feminino tendem a apresentar maiores complicações advindas da infecção dentária não tratada celeremente (Mathew et al., 2012).

Assim, o caso relatado vai ao encontro dos achados de Mathew et al., (2012), uma vez que a paciente se enquadra no perfil de pacientes mais acometidos pelas infecções. Ademais, o mesmo é corroborado também pelo acometimento grave dos espaços fasciais em paciente jovem e do gênero feminino sendo atendida em ambiente hospitalar, algo também demonstrado por Seppanen et al. (2011), que apontaram grande parte das infecções odontogênicas necessitarem de cuidados em ambientes hospitalares, com cerca de quinze dias de internação, sendo a faixa de idade média dos pacientes de 20 a 30 anos.

As infecções odontogênicas apresentam características clínicas e sintomatológicas que determinam o avanço da condição, entre estas, a dor, o edema, a febre, o trismo, a dispneia, a disfagia, a dislalia, a prostração, evoluindo se não tratado a tempo, para abscesso, celulites, trombose e até sepse, podendo levar o paciente a óbito (Ogle, 2017). A infecção pósoperatória pode evoluir lentamente e manifestar-se dias após a exodontia, ou, rapidamente, em menos de 24 horas após o procedimento cirúrgico, tornando-se mais graves, geralmente alastradas por uma debilidade sistêmica e com maiores chances de levar a óbito, como no caso relatado e corroborado pela literatura (Rios et al., 2021).

A gravidade de cada caso é determinada diante do estágio da infecção e da capacidade de resposta imunológica do hospedeiro frente a ela (Diniz et al., 2021). Tal fato foi demonstrado no caso acima, apesar de jovem e a princípio sem alterações sistêmicas, a resposta da paciente após a exodontia e o relato de pericoronarite prévia foi muito ruim, em pouco tempo a paciente teria uma evolução desastrosa. O sistema imune foi o ponto chave para analogia da resposta local inadequada e sistêmica deficitária.

As causas das infecções odontogênicas já são estabelecidas, tais como, a cárie dentária, as lesões endodônticas não tratadas ou com tratamento inadequado, infecções dento-alveolares prévias, pericoronarite, periodontites, granulomas e cistos apicais e complicações pós-cirúrgicas (Santos et al., 2020; Figueiredo et al., 2021). Dentre estas, ressalta-se a pericoronarite, quadro que a paciente apresentou previamente exodontia e a internação hospitalar, se não tratada previamente a exodontia pode sim relacionar-se a quadros sérios de infecção, tais como a Angina de Ludwig, de evolução rápida e potencialmente fatal (Guzman-Letelier et al., 2017; Rios et al., 2021).

Diante disso, é imprescindível diagnosticar a causa, a gravidade e sobretudo, o estado dos mecanismos de defesa do hospedeiro para um tratamento eficaz. Por mais que o procedimento não seja complexo, o tratamento da pericoronarite, embora bem estabelecido pela literatura, ainda assim vê-se muitas situações de negligência da terapêutica. A exodontia por si só não resolve o quadro da pericoronarite e sim pode levar a infecções pós-exodontia de difícil resolução. Amiúde, o mais complexo é o diagnóstico sistêmico, especialmente em pacientes jovens sem conhecimento de doenças preexistentes (Fonseca et al., 2020). O tratamento consiste em uma terapia básica: remoção da causa, drenagem cirúrgica e antibioticoterapia empírica, inegavelmente essenciais, entretanto, nunca negligenciando a atenção a fatores sistêmicos relacionados diretamente à incapacidade de controlar a infecção (Faverani et al., 2020).

A resistência sistêmica do paciente é, possivelmente, o fator mais importante para um bom resultado. Corroborando com o caso exposto, a infecção apresenta melhoras frente ao tratamento convencional, mas sobretudo, à resposta do organismo frente ao quadro. Em decorrência da gravidade do caso apresentado parece ser evidente que o tratamento prévio da pericoronarite diminuiria as possíveis complicações da exodontia dos terceiros molares. (Machado et al., 2004; Eller et al., 
2018).

\section{Conclusão}

Diante dos dados literários e com base no caso exposto, pode-se salientar a importância no diagnóstico inicial de uma infecção odontogênica e suas consequências na evolução do quadro clínico. O cirurgião dentista deve ter domínio das características de uma infecção de origem odontogênica e seus tratamentos, devendo discernir quando é necessária uma intervenção a nível ambulatorial ou hospitalar. É imprescindível, também, conhecimento sobre as medicações de uso empírico e a técnica cirúrgica empregada, pois, quanto antes realizado o tratamento, menor será a complicação na evolução do caso.

Ademais, é de grande importância atentar-se para os meios de prevenção, ressaltando a importância de investimentos nas ações da atenção básica dentro da odontologia preventiva, que são indispensáveis para evitar a cárie, a doença periodontal, bem como o tratamento de condições previas desfavoráveis, evitando assim, que estas evoluam para uma infecção odontogênica.

Uma vez que o relato de caso é único se faz necessário mais estudos acerca do tema afim de constatar a eficácia da terapêutica empregado e o sucesso em longo prazo.

\section{Agradecimentos}

O presente trabalho foi realizado com apoio da Fundação Universidade Federal de Mato Grosso do Sul -UFMS/MEC -Brasil.

\section{Referências}

Akinbami, B. O., \& Godspower, T. (2014). Dry Socket: Incidence, Clinical Features, and Predisposing Factors. International Journal of Dentistry. 1-7. $10.1155 / 2014 / 796102$.

Brito, T. P., Hazboun, I. M., Fernandes, F. L., Bento, L. R., Zappelini, C. E. M., Chone, C. T., et al., (2017). Abscessos cervicais profundos: estudo de 101 casos. Brazilian Journal of Otorhinolaryngology. 83(3).

Diniz, D.A, Silva, A. L. I., Angelim, L. V., Mendonça, T. L. R., Nascimento, V. H. S., Silva, C. C. G., Gonçalves, K. K. N., Souza Júnior, F. A., Cunha, J. S., \& Carneiro, S. C. S. (2021). Odontogenic infection in deep cervical spaces: diagnosis and treatment. Research, Society and Development. 10(13): e365101321094. 10.33448/rsd-v10i13.21094.

Eller, M. M. L., Franco, G. A. B. P., Ramos, J. D. F., Oliveira, I., Lacerda, L. F., Furtado, M. P., Simão, N. R., \& Silva, J. S. (2018). Infecções odontogênicas e sua relação com a resposta imunológica. IV Seminário Científico da FACIG e II Jornada de Iniciação Científica da FACIG.

Faverani, L. P., Ferreira, G. R., Garcia Junior, I. R., Souza, F. A., Ibrahim, G. M. F., \& Gaetti Jardim, E. C. (2020). Tratamento cirúrgico de abscesso odontogênico em nível hospitalar. Archives of Health Investigation. 9(4): 382-384. https://doi.org/10.21270/archi.v9i4.4740

Figueiredo, E. L., Figueiredo, E. L., Silva, C. P., Silva, M. E. L., Peixoto, L. S. F., Araújo, H. T., Leão, J. C., \& Carvalho, A. A. T. (2021). Complex odontogenic infections: a literature review. Research, Society and Development. 10(9): E20310917668. 10.33448/rsd-v10i9.17668

Fonseca, E. L. G., Francisco, M. A., Santos, M. A. B. S., Lira, J. S., Tenório, L. F. et al. (2020). Odontogenic infections, from etiology to treatment: a literature review. Brazilian Journal of Development. 6(7): p.44396-44407

Friedman, J. W. (2007). The Prophylactic Extraction of Third Molars: A Public Health Hazard. American Journal of Public Health. 97 (9): $1554-1559$.

Galvão, E. L., Silveira, E. M., Oliveira, E. S., Cruz, T. M. M., Flecha, O. D., Falci, S. G. M., \& Gonçalves, P. F. (2019). Associação entre a posição do terceiro molar mandibular e a ocorrência de pericoronite: uma revisão sistemática e metanálise. Archives of Oral Biology. 107:104486. 10.1016/j.archoralbio.2019.104486.

Guzman-Letelier, M., Crisosto-Jara, C., Diaz-Ricouz, C., Peñarrocha-Diago, M., \& Peñarrocha-Oltra, D. (2017). Severe odontogenic infection: An emergency. Case report. Journal of Clinical and Experimental Dentistry. 1;9(2):e319-e324. 10.4317/jced.53308.

Lima, F. G. G. P., Rios, L. G. C., Cunha, L. T. M. Q., Rocha, F. S., \& Batista, J. D. (2018). Clinical-surgical approach to complex infection in maxillofacial region: a case report. Revista Odontológica do Brasil Central. 27(81): 112-116

Machado, P. R. L., Araújo, M. I. A. S., Carvalho, L., \& Carvalho, E. M. (2004). Mecanismos de resposta imune às infecções. Anais brasileiros Dermatol. 79(6):647-664. 
Research, Society and Development, v. 10, n. 16, e560101624144, 2021 (CC BY 4.0) | ISSN 2525-3409 | DOI: http://dx.doi.org/10.33448/rsd-v10i16.24144

Mathew, G. C., Ranganathan, L. K., Gandhi, S., Jacob, M. E., Singh, I., Solanki, M., \& Bither, S. (2012). Infecções odontogênica do espaço maxilofacial em um centro de atendimento terciário no norte da Índia: um estudo retrospectivo de cinco anos. International Journal of Infectious Diseases. 16 (4): 296-302.

Ogle, O. E. (2017). Odontogenic Infections. Dental Clinics of North America. 61(2), 235-252.

Pitekova, L., Satko, I., \& Novotnakova, D. (2010). Complications after third molar surgery. Bratisl Lek Listy. 111(5): $296-298$.

Rios, B. R., Coelho, B. M., Monteiro, N. G., Santos, J. M. F. E., Silva, M. C., Barbosa, S., Silva, W. P. P., Martins, E. P., Melucci Filho, A., Souza, F., Ávila Magro Filho, O., Bassi, A. P. F., Ponzoni, D., Garcia-Júnior, I. R. R., \& Faverani, L. P. (2021). The importance of multidisciplinarity in surgical and supportive conduct in severe odontogenic infection: Case Report. Research, Society and Development. 10(5): e13810514846. 10.33448/rsd-v10i5.14846.

Santos, G. H. S., Lima, J. P., Armas, C. A. M., \& Pimenta, Y. S. (2020). Manejo de pacientes diagnosticados com infecções odontogênicas: revisão de literatura. Brazilian Journal of Development. 6(12): 95289-95298.

Seppänen, L., Lemberg, K. K., Lauhio, A., Lindqvist, C., \& Rautemaa, R. (2011). Is dental treatmentof an infected tooth a risk factor for locally invasive spread of infection? Journal Oral Maxillofacial Surgery. 69(4): 986-993.

Velasco, M. I., \& Soto, N. R. (2012). Principios para el tratamiento de infecciones odontogénicas con distintos niveles de complejidad. Revista Chilena de Cirurgía. 2012; 64(6): 586-598.

Wehr, C., Cruz, G., Young, S., \& Fakhouri, W. D. (2019). An Insight into Acute Pericoronitis and the Need for an Evidence-Based Standard of Care. Dental Journal (Basel). 7(3): 88. 